Reprod. Nutr. Dévelop., 1980, 20 (1 B), 271-276.

\title{
Sécrétion, métabolisme et transport de l'aldostérone dans la période néonatale chez le Cobaye
}

par J. GIRY, P. DELOST

Laboratoire de Physiologie Animale ef E.R.A. C.N.R.S.

Endocrinologie du Développement

Université de Clermont-Ferrand

Ensemble Scientifique des Cézeaux

B. P. 45, 63170 Aubière.

Summary. Secrefion, protein-binding and metabolic clearance rate of aldosterone in the guinea-pig during the neonatal period.

Aldosterone-binding to plasma proteins was determined in the guinea-pig by equilibrium dialysis, and the metabolic clearance rate (MCR) of aldosterone was calculated from experiments in which the labelled hormone was infused at a constant rate into the circulation during the neonatal period; no specific aldosterone-binding protein was found in the plasma. The hormone was weakly bound to the albumine. The MCR of aldosterone (1/day/100 g body weight) was unchanged from birth to day 8 , and its values were similar to those found in adults. Since there was no modification in aldosterone-binding to plasma proteins and no change in the MCR from birth to day 3 , the high plasma aldosterone concentrations in the guinea-pig were probably related to intense adrenal activity.

\section{Introduction.}

Les concentrations plasmatiques en aldostérone sont élevées à la naissance, dans le sang du cordon chez le nouveau-né humain (Beitins et al., 1972 ; Katz ef al., 1974 ; Tochigi, 1976 ; Dillon et al., 1976 ; Godard et al., 1976 ; Siegler et al., 1977), ainsi que dans le sang périphérique du poulain (Giry et al., 1979), du cobaye (Giry et Delost, 1974,1977 ) et de la souris (Dalle et al., 1978). Il est peu probable que ces fortes aldostéronémies soient d'origine maternelle, car Bayard et al. (1970) ont montré que les surrénales du fœtus humain, en fin de grossesse, sécrètent 80 p. 100 de l'aldostérone détectée dans le sang du cordon, alors que Giry et Delost (1979) observent que les surrénales du fœtus de cobaye, au 67 e jour de la gestation (terme 68 jours) sécrètent au moins 65 p. 100 de l'hormone minéralocorticoïde mesurée dans le compartiment fotal. Chez le cobaye nouveau-né, l'évolution de l'aldostéronémie est parallèle à celle de la teneur surrénalienne en aldostérone : elle est caractérisée par un pic à la $12 \mathrm{e}$ heure, suivi d'une chute qui se poursuit jusqu'au $3^{e}$ jour néonatal (Giry et Delost, 1977). Si les fortes 
valeurs dans le sang à la $12^{e}$ heure sont probablement en relation avec une intense stimulation de la surrénale néonatale, comme en témoigne le contenu glandulaire à ce stade, la chute brutale de l'aldostéronémie dans les heures qui suivent pourraif être due soit à la mise en route ou à une augmentation du catabolisme hépatique, soit à une modification de la liaison de l'hormone aux protéines plasmatiques, exposant celle-ci à l'action des enzymes responsables du catabolisme. Ce sont ces deux éventualités que nous avons étudiées dans ce travail, grâce à la détermination du taux de clairance métabolique et du pourcentage de liaison de l'aldostérone aux protéines plasmatiques au cours de la période néonatale chez le cobaye.

\section{Matériel et méthodes.}

A. Animaux. - Les cobayes de souche Dunkin-Hartley proviennent de la reproduction régulièrement entretenue au Laboratoire. Les femelles pubères (600-700 g) sont mises en présence du mâle pendant $24 \mathrm{~h}$ au cours de l'œstrus. Celles qui sont reconnues pleines par palpation sont isolées jusqu'à la mise-bas (terme 68 jours). Tous les animaux ont un libre accès à la nourriture (Aliment complet, UAR) et à l'eau de boisson.

a) Animaux sacrifiés pour l'étude de la liaison de l'aldostérone aux protéines plasmatiques. Les nouveau-nés sont décapités moins de $30 \mathrm{~s}$ après leur sortie de la cage, aux stades suivants : au moment de l'expulsion $(0 \mathrm{~h})$, puis entre 9 et $11 \mathrm{~h}$ aux $2 \mathrm{e}, 3 \mathrm{e}, 4^{\mathrm{e}}, 5^{\mathrm{e}}$, $6 \mathrm{e}, 7^{\mathrm{e}}, 8^{\mathrm{e}}$ et $20^{\mathrm{e}}$ jours. Après centrifugation du sang recueilli au cou, le plasma est aussitôt congelé à $-35^{\circ} \mathrm{C}$.

b) Animaux opérés pour l'étude du taux de clairance méfabolique. Les nouveau-nés, âgés de $6 \mathrm{~h}, 12 \mathrm{~h}, 3$ jours, 5 jours, 8 jours et 20 jours sont anesthésiés au chloral $(30 \mathrm{mg}$ par $100 \mathrm{~g}$ de poids corporel). Des cathéters sont introduits dans la veine jugulaire droite et la carotide gauche. Une solution de $\mathrm{D}-\left[1,2,6,7-{ }^{3} \mathrm{H}\right]$-aldostérone purifiée avant l'emploi par chromatographie sur papier, est perfusée en continu dans la jugulaire. Les échantillons de sang sont prélevés à partir de la carotide.

\section{B. Techniques.}

a) La technique de dialyse à l'équilibre sur sephadex de Pearlman ef Crepy (1967) adaptée au Laboratoire à l'étude de la liaison de l'aldostérone aux protéines plasmatiques par Pradier (1979) a permis de déterminer le pourcentage de liaison de l'hormone aux albumines. La quantité de plasma nécessaire à l'estimation de la liaison étant importante, chaque dialyse a été effectuée à partir d'un pool plasmatique groupant 2 à 4 animaux.

b) Perfusions. La D-[1,2,6,7-3H] aldostérone, d'activité spécifique $80-105 \mathrm{Ci} / \mathrm{mmole}$ (New England Nuclear), esł purifiée par chromatographie sur papier, puis diluée dans une solution de glucose 5 p. 100 à une concentration de $5.10^{6}$ d.p.m./ml ; le perfusat est placé dans une seringue de $5 \mathrm{ml}$ fixée à une pompe dont le débit est réglé à $0,62 \mathrm{ml} / \mathrm{h}$. Le taux de perfusion de $\mathrm{D}-\left[1,2,6,7-{ }^{3} \mathrm{H}\right]$ aldostérone est mesuré après collection de 3 effluents de 10 min à partir de la pompe. 
Quatre échantillons de sang de $0,1 \mathrm{ml}$ sont prélevés par l'intermédiaire du cathéter carotidien à $90,100,110,120$ min après le début de la perfusion. Après centrifugation, le plasma, auquel on ajoute $15 \mu \mathrm{g}$ d'aldostérone froide et 1,0 $\mathrm{ml}$ d'eau bidistillée, est extrait par 3 fois $5 \mathrm{ml}$ de dichlorométhane à $0{ }^{\circ} \mathrm{C}$. Les extraits sont regroupés, évaporés à sec sous azote, puis chromatographiés sur papier dans le système de solvants Bush B5. Après élution au méthanol, chaque échantillon est concentré sous azote, puis mis en solution dans $10 \mathrm{ml}$ de liquide scintillant et placé dans le compteur à scintillation.

Le taux de clairance métabolique (TCM) est défini comme étant le rapport du taux de perfusion de l'hormone en d.p.m./min sur la concentration plasmatique de l'hormone radioactive à l'équilibre en d.p.m./ml. Ełant donné que les valeurs du TCM de l'aldostérone et de la liaison sont pratiquement identiques chez le mâle et chez la femelle, nous avons groupé les 2 sexes pour la moyenne. Les moyennes des valeurs sont données avec erreur-type et la validité statistique des différences entre les moyennes est calculée par le test de Fisher-Student.

\section{Résultats.}

a) Pourcentage d'hormone liée au cours de la période néonatale (fig. 1). - Nous n'avons pu mettre en évidence une protéine spécifique de liaison de l'aldostérone chez le cobaye ; cette hormone est uniquement liée aux albumines. Le pourcentage d'hormone liée aux albumines, faible à la naissance (21.4 \pm 2 p. 100) augmente régulièrement jusqu'au $20^{\mathrm{e}}$ jour posłnatal $(31.5 \pm 1.5 \mathrm{p}$. 100). La différence entre ces deux stades extrêmes est hautement significative $(P<0.001)$. Au $20 \mathrm{e}$ jour, ce paramètre de liaison n'est pas significativement différent de celui que l'on observe chez l'adulte (35.4 \pm 3.7 p. 100).

FIG. 1. - Evolution du pourcentage de lioison de laldostérone aux albumines plasmatiques au cours de la période néonatale chez le Cobaye.

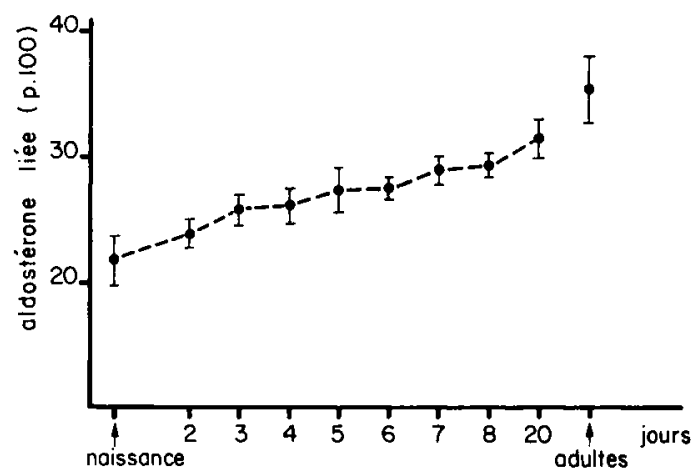

b) TCM de l'aldostérone au cours de la période néonatale (fig. 2). - Exprimé en litres par $24 \mathrm{~h}$, le TCM est stable de la $6^{\mathrm{e}}$ heure $(2.1 \pm 0.1)$ au $5^{\mathrm{e}}$ jour post-natal $(2.4 \pm 0.8)$; puis il augmente significativement entre le $5 \mathrm{e}$ jour et le $8 \mathrm{e}$ jour $(3.3 \pm 0.3$; $0.02<\mathbf{P}<0.05)$. La valeur la plus élevée s'observe au $20 \mathrm{e}$ jour $(8.9$ 土 0.4$)$; la différence entre la $6^{\mathrm{e}}$ heure et le $20^{\mathrm{e}}$ jour, ainsi que celle entre le $8^{\mathrm{e}}$ et le $20^{\mathrm{e}}$ jour sont hautement significatives $(P<0.001)$. 
Exprimé en litres par $24 \mathrm{~h}$ et pour $100 \mathrm{~g}$ de poids corporel, le TCM ne varie pas de la 6 e heure au $8 \mathrm{e}$ jour ; il s'élève entre le $8 \mathrm{e}$ et le $20^{\mathrm{e}}$ jour pour atteindre une valeur significativement différente de celle observée au $8 \mathrm{e}$ jour $(P<0.001)$.

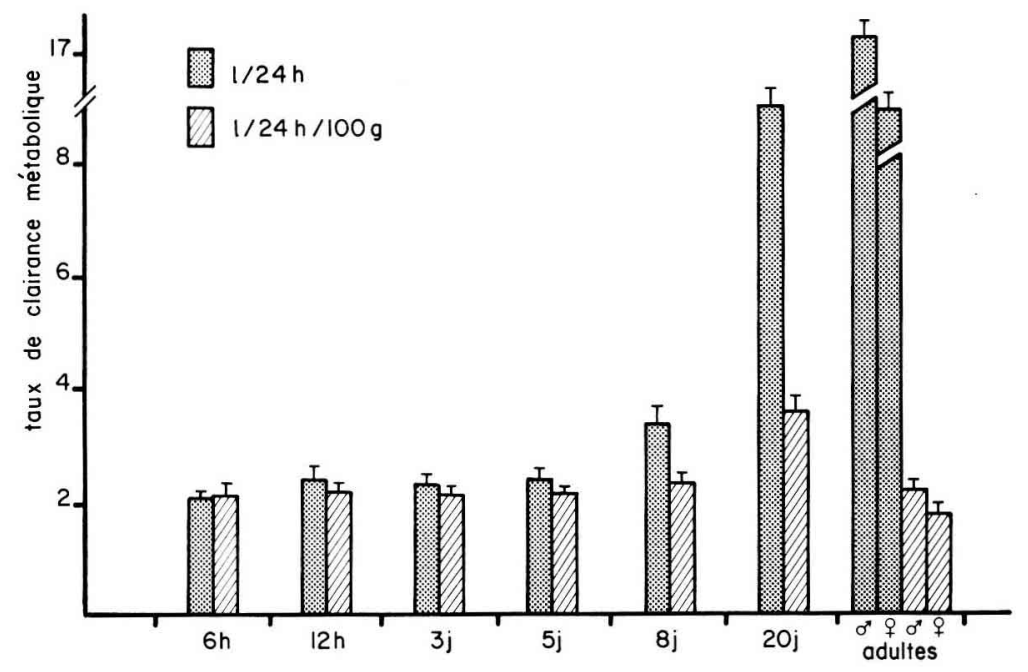

FIG. 2. - Evolution du taux de clairance métabolique (TCM) de l'aldostérone au cours de la période néonatale chez le Cobaye.

\section{Discussion.}

A notre connaissance, la liaison de l'aldostérone aux protéines plasmatiques n'a pas été étudiée jusqu'à maintenant chez l'animal. Dans l'espèce humaine, l'hormone sécrétée dans la veine surrénalienne est rapidement liée à la surface des globules rouges et aux protéines plasmatiques (Holzbauer et Vogt, 1961). Parmi les protéines du plasma, l'albumine a d'abord été reconnue comme étant la principale protéine de liaison de l'hormone minéralocorticoïde (Daughaday et al., 1961 ; Tait et Burstein, 1964), puis comme l'aldostérone avait une forte affinité pour une globuline, Meyer et al. (1961), Westphal (1966) et Zager ef al. (1976) ont suggéré qu'il s'agissaił de la transcortine. Ce n'est que tout récemment que Richardson et al. (1977) ont mis en évidence une « aldosterone-binding-globulin » ( $A B G$ ) dont les caractéristiques physicochimiques sont différentes de celles de la transcortine. La technique de dialyse à l'équilibre sur sephadex qui différencie une liaison spécifique d'une liaison non spécifique nous a permis de constater que chez le cobaye, au cours de la période néonatale aussi bien que chez l'adulte, il n'existe pas de liaison spécifique de l'aldostérone aux protéines plasmatiques. Seule une liaison lâche associe l'hormone et l'albumine dans cette espèce. La liaison, à la naissance, est extrêmement faible, puisqu'elle esł à peine supérieure à 20 p. 100 . Elle esí identique à celle que l'on détermine dans le compartiment fœilal en fin de gestation (observations non publiées). L'augmentation régulière du pourcentage de liaison à l'albumine que l'on observe de la naissance au $20^{\circ}$ jour, stade auquel les valeurs de l'adulte sont atteintes, pourrait être le simple reflet de l'augmentation de la concentration des protéines dans le plasma, puisque celle-ci passe 
de $41 \mathrm{~g} / \mathrm{l}$ à la naissance à $50 \mathrm{~g} / \mathrm{l}$ au $10^{\circ}$ jour ; par ailleurs elle ne semble pas représenter une prolection vis-à-vis du catabolisme hépatique puisque le TCM de l'aldostérone augmente fortement au 20 jour. Les conditions opératoires nous ont obligé à anesthésier le cobaye pour mesurer le TCM de l'aldostérone dans la période néonatale. Il est difficile d'exclure une éventuelle action de l'anesthésie sur le TCM aux stades étudiés ; toutefois Khaldoun et al. (1977) ont montré que chez le cobaye, le TCM de l'aldostérone étaił identique au stade de 50 jours chez l'animal vigile et chez l'animal anesthésié.

Exprimé en litres par $24 \mathrm{~h}$, le TCM est faible de la naissance au 5 e jour néonatal ; sa valeur est environ 8 fois inférieure à celle du mâle adulte âgé de 6 mois et 6 fois inférieure à celle de la femelle adulte de même âge, animaux chez lesquels Khaldoun et al. (1977) ont mis en évidence un dimorphisme sexuel du TCM de l'aldostérone. L'élévation du TCM $(1 / 24 \mathrm{~h})$ entre le $5^{\mathrm{e}}$ et le $8^{\mathrm{e}}$ jour post-natal (fig. 2) doit être en relation avec l'augmentation du poids du corps $(+15 \mathrm{p}$. 100) qui se produit au cours de cette période car si l'on exprime le TCM en $1 / 24 \mathrm{~h} / 100 \mathrm{~g}$ de poids corporel, ce paramètre ne varie pas. Il est intéressant de noter que le TCM $(1 / 24 \mathrm{~h} / 100 \mathrm{~g})$ le plus élevé est observé au 20 jour néonatal, stade où les valeurs de l'aldostéronémie sont les plus basses (Khaldoun et al., 1977).

Sur la base des résultats de Weldon et al. (1967) qui rapportent des taux de sécrétion d'aldostérone constamment bas chez le nouveau-né humain de moins de $170 \mathrm{~h}$, Bayard ef Boulard (1973) avaient émis l'hypothèse selon laquelle, chez le nouveau-né, l'aldostérone serait métabolisée plus lentement que chez l'adulte du fait de l'immaturité du système enzymatique responsable du catabolisme. II ne semble pas que cela soił le cas chez le cobaye car le TCM $(1 / 24 \mathrm{~h} / 100 \mathrm{~g})$ n'est pas significativement différent de celui de l'adulte. De plus, la chute brutale de l'aldostéronémie qui se produit entre la $12^{\mathrm{e}} \mathrm{h}$ et le $3^{\mathrm{e}}$ jour dans cette espèce $n^{\prime}$ est pas le reflet d'une modification du catabolisme de l'hormone dans la mesure où, quel que soit le mode d'expression du TCM, ce paramètre ne varie pas. Dans les 3 premiers jours après la naissance chez le cobaye, la stabilité du taux de clairance métabolique ef l'absence de modification nette du pourcentage de liaison de l'hormone à la protéine vectrice, montrent que la concentration plasmatique est le reflet de l'activité de la surrénale, comme en témoigne l'évolution du contenu surrénalien en aldostérone (Giry et Delost, 1977). D'autre part, les valeurs élevées de l'aldostéronémie et du contenu glandulaire en aldostérone à la $12^{\mathrm{e}} \mathrm{h}$, traduisent une intense stimulation de la glande ; mais la nature ef l'origine du ou des facteurs trophiques restent à déterminer.

$5^{e}$ Réunion du groupe Développement I.N.R.A., Clermont-Ferrand/Theix, 17-18 mai 1979.

\section{References}

BAYARD F., ANCES I. G., TAPPER A. J., WELDON V. V., KOWARSKI A., MIGEON C. J., 1970. Transplacental passage and fetal secretion of aldosterone. J. clin. Invest., 49, 1389-1393.

BAYARD F., BOULARD C., 1973. La sécrétion d'aldostérone chez le fœetus et le nouveau-né, 34-42. Hormones ef régulations métaboliques, Masson et Cie, Paris.

BEITINS I. Z., BAYARD F., LEVITSKY L., ANCESI. G., KOWARSKI A., MIGEON C. J., 1972. Plasma aldosterone concentration at delivery and during the newborn period. J. clin. Invest., 51, 386394. 
DALLE M., GIRY J., GAY M., DELOST P., 1978. Perinatal changes in plasma and adrenal corticosterone and aldosterone concentrations in the mouse. J. Endocr., 76, 303-309.

DAUGHADAY W. H., HOLLOSZY J., MARIZ I. K., 1961. Binding of corticosteroids by plasma proteins. VI. The binding of cortisol and aldosterone by corticosteroid-binding globulin and by the estrogen-induced binding system of plasma. J. clin. Endocrinol. Metab., 21, 53-57.

DILLON M. J., GILLIN M., RYNESS J. M., DE SWIET M., 1976. Plasma renin activity and aldosterone concentration in the human newborn. Arch. Dis. Child., 51, 537-540.

GIRY J., DELOST P., 1974. Evolution de l'aldostéronémie du fœłus et du nouveau-né de cobaye au cours de la période périnatale. C. R. Acad. Sci. Paris, Sér. D, 279, 2087-2090.

GIRY J., DELOST P., 1977. Changes in the concentrations of aldosterone in the plasma and adrenal glands of the fotus, the newborn and the pregnant guineapig during the perinatal period. Acta endocr., 84, 133-141.

GIRY J., DELOST P., 1979. Placental transfer of aldosterone in the guinea-pig during late pregnancy. J. Steroid Biochem., 10, 541-547.

GIRY J., KHALDOUN M., TOURNAIRE C., BARLET J. P., MARTIN-ROSSET W., DELOST P., 1979. L'aldostérone chez la jument en fin de gestation et chez le poulain au cours de la période néonatale. J. Physiol. (Paris) (sous presse).

GODARD C., GAILLARD R., VALLOTTON M. B., 1976. The renin-angiotensinaldosterone system in mother and fetus at term. Nephron, 17, 353-360.

HOLZBAUER M., VOGT M., 1961. Corticosteroids in plasma and cells of adrenal venous blood. J. Physiol. (Lond.), 157, 137-156.

KATZ F. H., BECK P., MAKOWSKI E. L., 1974. The renin-aldosterone system in mother and fetus at term. Am. J. Obstef. Gynecol., 118, 51-55.

KHALDOUN T., GIRY J., DELOST P., 1977. Concentration plasmatique et taux de clairance métabolique de l'aldostérone chez le cobaye au sevrage, à la puberté et à l'âge adulte. C. R. Soc. Biol., 171, 784-790.

MEYER C. J., LAYNE D. S., TAIT J. F., PINCUS G., 1961. The binding of aldosterone to plasma proteins in normal, pregnant, and steroid-treated women. J. clin. Invest., 40, 1663-1671.

PEARLMAN W. H., CREPY O., 1967. Steroid protein interaction with particular reference to testosterone binding by human serum. J. biol. Chem., 242, 182-189.

PRADIER P., 1979. Liaison de la testostérone, de la dihydrotestostéroneà et de l'aldostérone chez le foctus ef le nouveau-né de cobaye pendant lo période périnatale et chez la mère pendant la gestation. Th. $3^{\text {e }}$ Cycle, Clermont-Ferrand, France, 138 pp.

RICHARDSON K. S. C., NOWACZYNSKI W., GENEST J., 1977. Specific aldosterone-binding proteins in human plasma : partial characterisation. J. Steroid Biochem., 8, 951-957.

SIEGLER R. L., CROUCH R. H., HACKETT T. N., WALKER M., JUBIZ W., 1977. Potassium-reninaldosterone relationships during the first year of life. J. Pediatr., 91, 52-55.

TAIT J. F., BURSTEIN S., 1964. In vivo studies of steroid dynamics in man, 441-557. In PINCUS G., THIMAN K. V., ASTWOOD E. B., The hormones, Vol. 5, Acad. Press, New York.

TOCHIGI B., 1976. Studies on aldosterone concentration of maternal, fetal and neonatal plasma during pregnancy, parturition and neonatal period. Nichidai lgaku Zasshi., 35, 857-868.

WELDON V. V., KOWARSKI A., MIGEON C. J., 1967. Aldosterone secretion rates in normal subjects from infancy to adulthood. Pediatrics, 39, 713-723.

WESTPHAL U., DE VENUTO F., 1966. Steroid-protein interactions. XI. Electrophoretic characterisation of corticosteroid-binding proteins in serum of rat, man and other species. Biochem. biophys. Acfo, 115, 187-193.

ZAGER P. G., BURTIS W. J., LUETSCHER J. A., DOWDY A. J., SOOD S., 1976. Increased plasma protein binding and lower metabolic clearance rate of aldosterone in plasma of low cortisol concentration. J. clin. Endocrinol. Metab., 42, 207-214. 